\title{
Porous Resin Catalyst with Strong Acidity for Hydration Reaction of Butane and Its Catalytic Performance
}

\author{
Cai Jian-guo, Shi Hong-yan, Zhu Zhi-rong, Shi Xiao-li \\ Jiangsu Hyper Functional Materials Co. Ltd., Suzhou, China
}

\section{Email address:}

caijg@jshaipu.com (Cai Jian-guo), shihy@jshaipu.com (Shi Hong-yan), zhuzhirong@yahoo.com (Zhu Zhi-rong), admin@jshaipu.com (Shi Xiao-li)

\section{To cite this article:}

Cai Jian-guo, Shi Hong-yan, Zhu Zhi-rong, Shi Xiao-li. Porous Resin Catalyst with Strong Acidity for Hydration Reaction of Butane and Its Catalytic Performance. Science Discovery. Vol. 4, No. 2, 2016, pp. 137-141. doi: 10.11648/j.sd.20160402.23

Received: April 29, 2016; Accepted: May 17, 2016; Published: May 19, 2016

\begin{abstract}
With nano-alumina modified by the fat acid as an inorganic template for making pores, a new kind of porous resin particle with the large surface was synthesized by emulsion polymerization. Moreover, the sulfonated polystyrene resin was fluorinated to increase its acid strength up to Ho 8.6 and the acid amount of $4.1 \mathrm{mmol} / \mathrm{g}$. The ratio of butane to water was increased by packing the catalytic resin with the fixed beds of four stages. Under reaction temperature of $168-174^{\circ} \mathrm{C}$, butane to water ratio of $2 \mathrm{~mol} / \mathrm{mol}$ and WHSV1.0 h-1, hydration reaction conversion was over $15 \mathrm{wt} \%$, with the butyl alcohol selectivity of $99 \mathrm{wt} \%$ and a good thermal stability.
\end{abstract}

Keywords: Butene Hydration, Pores, Acid Resin, Catalyst, Reaction Performance

\section{用于丁烯水合的多孔强酸性树脂催化剂及其反应性能}

\author{
蔡建国，石洪雁，朱志荣，石小丽 \\ 江苏海普功能材料有限公司, 苏州, 中国

\section{邮箱} \\ cai jg@jshaipu. com（蔡建国）， shihy@jshaipu. com（石洪雁），zhuzhirong@yahoo.com（朱志荣）， \\ admin@jshaipu. com（石小丽）
}

\begin{abstract}
摘要: 采用经脂肪酸改性处理的纳米氧化铝为无机致孔剂的硬模板支撑造孔方法, 以悬浮聚合方式合成了高比表面积 的多孔苯乙烯树脂颗粒; 在对它磺化酸功能化后进一步进行了氟化修饰, 使苯乙烯树脂固体酸的酸强度Ho显著上升可 达-8. 6、酸量为 $4.1 \mathrm{mmol} / \mathrm{g}$; 通过采用四段装填方式固定床反应工艺, 提高反应接触的水/丁烯反应物料比, 催化剂在 反应温度 $168^{-}-174^{\circ} \mathrm{C}$, 水与丁烯比 $2 \mathrm{~mol} / \mathrm{mol}$, 空速WHSV1.0 h-1条件下烯烃直接水合催化反应的丁烯转化率达到 $15 \mathrm{wt} \%$ 以上、脂肪醇产物选择性 $99 \mathrm{wt} \%$ 以上, 并具有较好的反应稳定性。
\end{abstract}

关键词: 丁烯水合, 孔道, 酸性树脂, 催化剂, 反应性能

\section{1. 引言}

目前德国、日本等工业发达的国家及中国相关的烯烃 水合反应主要技术有: 传统硫酸间接水合、杂多酸催化直 接水合和树脂催化直接水合三种工艺方法 [1 - 5]。
1）间接水合工艺过程包括酯化、水解、精馏和稀酸 浓缩4 段工序, 反应副产物为二仲丁基醚及丁烯低聚物等。 用硫酸 (浓度 $80 \%$ 左右) 吸收预处理过的主要含正丁烯的混 合C4 馏分, 生成丁基硫酸酯, 再水解得到仲丁醇水溶液, 经精馏可得仲丁醇。该技术缺点是设备腐蚀严重, 装置投 
资较大, 需消耗大量硫酸和碱原料, 产生的三废多, 正丁烯 单耗较高。目前，此方法在国际上已基本被淘汰或禁止使 用。

2) 酸催化直接水合反应器采用立式搅拌槽, 内装杂多 酸催化剂, 主要成份是钼磷酸, 并加入有机金属化合物添 加剂, 反应温度 $200 \sim 230^{\circ} \mathrm{C}$, 反应压力 $19.0 \mathrm{MPa}$ 。正丁烯 单程转化率 $5 \% \sim 10 \%$, 仲丁醇选择性大于 $97 \%$ 。此类工业装 置缺点是反应压力过高, 温度也较高, 催化剂有损耗, 综 合经济性不够理想。

3 ) 树脂催化直接水合反应器采用筒式, 内装固体磺酸 型阳离子树脂催化剂, 该工艺流程简单, 产品回收精制容 易, 不消耗硫酸, 设备不易腐蚀, 三废少。缺点是对原料正 丁烯要求高, 正丁烯反应单程转化率较低 (6-8\%), 目前中 国均为此类型工业生产装置。

普通的磺化苯乙烯氢型阳离子树脂具有均一的酸强 度 (相当于 $75 \%$ 的H $2 \mathrm{~S} 04$ ), 较大的酸量以及较好的稳定性, 目前已作为固体酸代替H $2 \mathrm{SO}$ 4广泛地应用于各种酯化、醚 化、苯酚烷基化、烯烃水合等各种酸催化反应中。Texaco 公司开发了用 “固体硫酸” 磺化树脂作为催化剂, 直接水 合制备醇的新工艺。在强酸性离子交换树脂催化剂作用下 正丁烯与水的反应是一种放热的质子催化反应。反应中存 在三相: 固相 (催化剂), 液相 (水), 超临界相 (有机物)。正 丁烯通过质子催化作用水合反应生成SBA, 正丁烯的三个 异构体的水合速度和反应热略有差异。由于 C4 烃与水几 乎不互溶, 因此反应是在三相 (固相催化剂、液相水和超临 界有机相) 条件下进行, 水过量有利于提高SBA 的选择性。 树脂催化直接水合工艺不消耗硫酸, 无酸中和步骤; 设备 无腐蚀 (不含反应器); 无大量废水生成; SBA选择性高达 $98 \%$ 。不足之处是正丁烯单程转化率低, 仅约 6-8\%, 装置丁 烯物料循环能耗较大, 故存在改进催化剂以提高正丁烯单 程转化率的迫切需求。

近二十多年来, 德国、日本、美国等发达工业国家一 直致力于研究开发环境友好的高效烯烃直接水合反应固 体酸催化剂和反应工艺技术, 以提高目前生产工艺装置效 率。基于催化反应的特点, 近年来研究主要涉及的固体酸 催化剂有分子篮催化剂、负载型杂多酸催化剂和新型酸性 树脂催化剂 $[6,7]$ 。虽然负载型杂多酸催化剂同时具有高 酸强度和高比表面积的优点, 在合适的制备条件下能够将 酸中心均匀的分散在载体的表面上, 且固定床反应工艺操 作使用方便。但是该类催化剂存在着活性中心易流失, 催 化剂的使用寿命不够等缺点。以往的研究结果显示, 纳米 HZSM-5 分子篮具有更好的反应活性和稳定性, 故被认为是 一种很有前途的水合催化剂。但是, 沸石分子筛作为烯烃 直接水合催化剂存在的主要缺点是因为其反应失活较快, 原料的水/烯比很高, 会造成较高的能耗与物耗。目前德 国、日本等研发的这些固体酸作为多相催化剂普遍存在易 失活、稳定性差等缺点, 综合技术经济性不够理想。

由上可见, 比较而言, 酸性树脂催化剂具有较好的丁 烯水合的反应性能, 设想通过创新的制备手段改进目前 工业上的树脂固体酸催化材料催化剂性能, 并采用新的 配套反应工艺的研究路线, 以实现丁烯水合技术突破性 的进步。

\section{2. 实验方法}

\section{1. 多孔杂化树脂合成方法}

采用苯乙烯为聚合单体 (含5.0 wt\%二乙烯苯为交联 剂)、 $1.0 \mathrm{wt} \%$ 过氧化二异丙苯为引发剂、5.0-20.0 wt\% 无机纳米粉末致孔剂混合均匀后组成油相部分, 在 $3.5 \mathrm{wt} \%$ 聚乙烯醇等分散剂辅助下制成水性悬浮液, 在反应温度 $80-82^{\circ} \mathrm{C}$ 和一定的摚拌速度条件下进行聚合反应 $6 \mathrm{~h}$, 以合 成粒径约为 $1.0 \mathrm{~mm}$ 的苯乙烯树脂颗粒; 上述合成的树脂颗 粒用 $3.0 \% \mathrm{HCl}$ 溶液浸泡、过滤并水洗, 除去粉末致孔剂后干 燥, 制备得到多孔苯乙烯树脂颗粒。

\section{2. 多孔树脂磺化酸功能化}

采用浓硫酸与 $50 \mathrm{wt} \%$ 发烟硫酸混合物为磺化剂, 加入 少量 $1.5 \%$ 硫酸银磺化催化剂, 并选择二氯乙烷等适当溶剂 作为树脂颗粒溶胀剂, 在反应温度 $90-110^{\circ} \mathrm{C}$ 和搅拌条件下 进行磺化反应 $8 \mathrm{~h}$; 并采用逐步水稀释去残余硫酸、水蒸汽 脱溶剂方法后处理, 获得磺酸功能化的多孔酸性树脂催化 剂。

\section{3. 树脂催化剂固定床反应}

采用自制的 2-4段串联多级固定床反应工艺，填装制 备的树脂催化剂共 $20 \mathrm{ml}$, 在反应温度 $160-170^{\circ} \mathrm{C}$, 水与丁烯 比 $2 \mathrm{~mol} / \mathrm{mol}$, 空速WHSV $1.0 \mathrm{~h}-1$ 条件下, 进行树脂催化剂 的烯烃直接水合催化反应评价, 气相色谱收集产物的组成, 计算丁烯转化率与丁醇产物选择性。

\section{3. 结果与讨论}

\section{1. 多孔聚苯乙烯磺酸树脂催化剂的制备}

现有大孔聚苯乙烯酸性树脂催化剂的制备分为树脂 苯乙烯树脂颗粒的合成和硫酸磺化的酸功能化二个步骤 进行制备。传统的大孔树脂苯乙烯树脂颗粒的制备均采用 苯乙烯单体、聚合引发剂、二乙烯苯交联剂、以及有机溶 剂致孔剂混合均匀后组成油相部分, 在表面活性分散剂辅 助作用下和一定的摚拌速度条件下形成水性悬浮液; 然后 在反应温度下进行聚合反应, 水性悬浮液的油相液滴中苯 乙烯聚合形成的微粒在有机溶剂致孔剂中不断析出, 众多 微粒集聚在一起形成 $1.0 \mathrm{~mm}$ 左右的树脂颗粒, 待聚合反应 完成将微粒之间的有机溶剂致孔剂蒸发除去, 从而形成树 脂颗粒内部的孔隙, 制得多孔聚苯乙烯颗粒。但是, 此方 法制备得到多孔苯乙烯树脂颗粒中孔道结构的形态与大 小尺寸难于精确控制, 造成孔径较大与比表面积偏小, 不 利于其作为多相催化的高分子固体酸催化剂。

近二十年来采用各种表面活性剂胶束作软质致孔模 板剂的方法已广泛应用于多种规则介孔分子篮材料的合 成中; 近年来人们又采用无机纳米粉体或高分子化合物粉 体分散在需反应合成或成型制备材料的前驱体中作为硬 质致孔模板剂, 在完成制备过程后, 将颗粒之间的致孔剂 通过焙烧分解或反应溶解除去后, 形成了需制备颗粒内部 的孔隙, 从而制得了可控孔径与孔容的多孔催化剂颗粒。 
在本论文工作的高分子聚苯乙烯树脂颗粒合成制备中, 借 鉴采用了无机硬质致孔模板剂的造孔方法: 即以苯乙烯为 聚合单体、二乙烯苯为交联剂、过氧化二异丙苯为引发剂、 与无机致孔剂混合均匀后组成油相部分, 在聚乙烯醇等分 散剂辅助下制成水性悬浮液, 在一定的反应条件下进行聚 合反应，合成杂化的苯乙烯树脂颗粒; 并采用盐酸的乙醇 /水溶液对获得的聚合树脂颗粒中纳米氧化物进行溶脱的 多孔化处理, 实现无机致孔剂硬模板支撑的造孔, 以合成 高比表面积与多孔化的苯乙烯树脂颗粒。

但是, 研究中发现如果直接加入纳米氧化铝, 无机纳 米粉末不易在水性悬浮液做油相部分中均匀分散, 不能制 成具有高比表面积的多孔苯乙烯树脂颗粒, 同时颗粒形状 不规整且强度差。为了克服这个由于无机纳米粉末表面亲 水性引起的混合分散问题, 通过试验发现采用一定量的碳 链脂肪酸（即4-6wt\%己酸）对纳米氧化铝进行表面性质的 化学吸附改性处理, 可以大幅度提高无机致孔剂组份的疏 水性, 实现了改性纳米氧化铝在油相中均匀分散, 从而获 得了颗粒形状较规整并含有丰富中孔一大孔的聚合树脂颗 粒。通过分散在油相中无机纳米氧化物致孔剂组份的用量 变化可以调控聚合树脂颗粒内中孔-大孔孔道构建的多孔 化范围（即孔容与比表面积）。采用物理吸附仪与BET流 动色谱法结合, 实现对多孔树脂催化剂孔结构进行了全面 的分析表证。试验结果表1显示了不同硬模板致孔剂用量 对合成树脂孔结构的影响。

表1 致孔剂用量对合成树脂孔结构的影响。

\begin{tabular}{llll}
\hline 纳米氧化物/单体 \% & 比表面积 $\mathrm{m}^{2} / \mathrm{g}$ & 平径孔径 $\mathrm{nm}$ & 孔容 $\mathrm{ml} / \mathrm{g}$ \\
\hline 0 & 41 & 7.5 & 0.32 \\
9.5 (未改性) & 77 & 28.9 & 0.50 \\
3.8 & 69 & 12.4 & 0.47 \\
7.1 & 90 & 15.6 & 0.55 \\
9.6 & 112 & 17.1 & 0.58 \\
13.7 & 141 & 20.3 & 0.63 \\
19.2 & 184 & 24.9 & 0.72 \\
\hline
\end{tabular}

由上表 1 结果可见, 将疏水性纳米氧化物组份混合均 匀后组成的悬浮聚合油相部分中纳米氧化物/单体的含量 为 $1 / 10$ 左右较为合适, 制得的多孔苯乙烯树脂颗粒具有较 高的比表面积与强度, 且颗粒形状规整。

为了将多孔苯乙烯树脂颗粒更好的酸功能化, 能作为 多孔树脂固体酸催化剂应用。试验选择考察了磺化反应处 理过程中的磺化剂硫酸浓度、磺化催化剂、溶胀剂对磺化 后树脂酸量的影响, 以控制苯乙烯树脂孔壁表面上的磺化 过程沿表面径向梯度扩散进行反应, 从而使获得的多孔磺 酸树脂具有更高的表面酸中心密度。参数优化选择试验中 发现磺化剂硫酸浓度条件对磺化树脂酸量的影响最为显 著。通过离子交换滴定法化学分析测定了多孔磺酸树脂催 化剂的酸量, 实验结果表明采用浓硫酸与 $50 \mathrm{wt} \%$ 发烟硫酸 混合物为磺化剂时, 可以获得较好多孔树脂磺酸化效果, 多孔树脂磺酸化后制备的催化剂酸量可达 $4.9 \mathrm{mmol} / \mathrm{g}$ 。

\section{2. 多孔磺酸树脂催化剂的卤化修饰改性}

从以往大量的针对丁烯水合反应合成脂肪醇的催化 研究总结中, 已基本明确了理想的水合催化反应固体酸催
化剂应具有的特征为: (1) 较高酸强度与总酸量（高反 应活性），（2）含有中孔/大孔的高比表面积、且酸中心 稳定分布在表面上（高脂肪醇产物选择性、低结焦失活速 率）。因此, 对苯乙烯磺酸树脂通过其苯环上卤化修饰是 增强树脂酸性的一个有效方法 [8-10]。

首先从氟化过程的安全性与可控性上考虑, 选择了将 苯乙烯磺酸树脂先用氯气氯化后, 再以稀释氟气作为氟化 剂进行氟取代反应的氟化方法; 试验优化了多级孔杂化磺 酸树脂氟化反应处理的工艺与条件获得了更强酸性的多 级孔氟化磺酸树脂固体酸; 同时采用先进的XPS电子能谱 表面分析技术与采用Hammett指示剂滴定法, 对氟化修饰 前后树脂固体酸的酸性与结构组成进行了测定分析, 具体 结果见图1与表2。

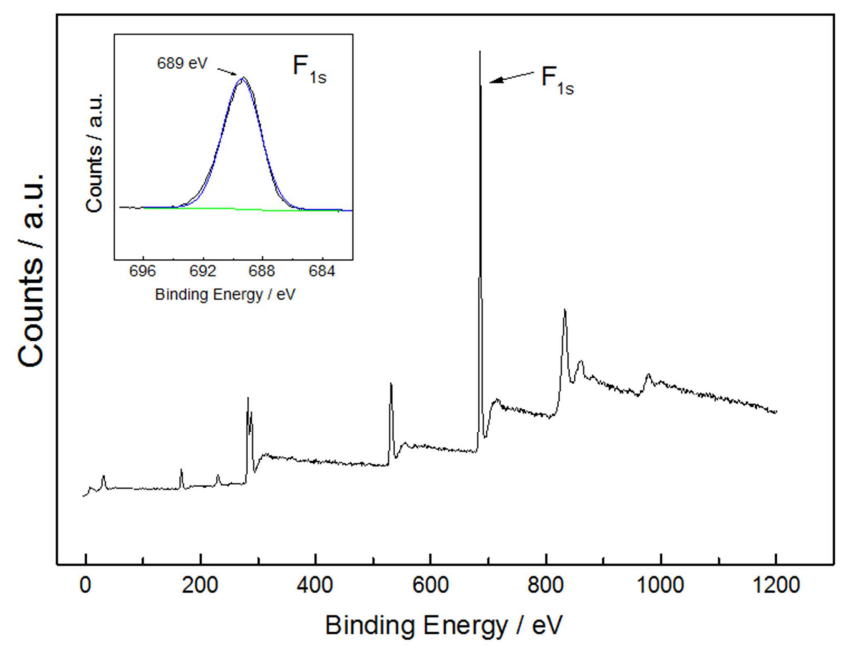

图1 氟化后磺酸树脂的XPS图谱。

表2 氟化磺酸树脂催化剂的酸性质。

\begin{tabular}{|c|c|c|c|c|c|}
\hline 催化剂 & $\begin{array}{l}\text { 多孔磺 } \\
\text { 酸树脂 }\end{array}$ & $\begin{array}{l}\text { 多孔氯 } \\
\text { 化磺 } \\
\text { 树脂 }\end{array}$ & $\begin{array}{l}\text { 多孔氟 } \\
\text { 化磺酸 } \\
\text { 树脂 }\end{array}$ & $\begin{array}{l}\text { 商品大 } \\
\text { 孔磺酸 } \\
\text { 树脂 }\end{array}$ & $\begin{array}{l}\text { 商品凝 } \\
\text { 胶磺酸 } \\
\text { 树脂 }\end{array}$ \\
\hline 酸强度Ho & -7.2 & -7.9 & -8.6 & -7.1 & -7.0 \\
\hline $\begin{array}{l}\text { 酸量 } \\
\mathrm{mmol} / \mathrm{g}\end{array}$ & 4.9 & 3.8 & 4. 1 & 4.5 & 4.7 \\
\hline
\end{tabular}

从图1氟化后磺酸树脂的XPS图谱上清晰可见, 氟化修 饰后苯乙烯树脂固体酸苯环结构组成上卤化 C-F键的存在。 由表3可见, 不同酸性树脂催化剂的酸强度大小值却存在 着较大的差异, 在氯化或氟化修饰后苯乙烯树脂固体酸的 酸强度Ho显著上升, 氟化修饰后苯乙烯树脂固体酸的酸强 度Ho高至-8. 6 (酸量下降主要由氯化或氟化修饰使苯乙烯 树脂增重所引起）。通常酸强度Ho在 -7.1 至 -7.3 左右相当 于 $75 \mathrm{wt} \%$ 的 $\mathrm{H} 2 \mathrm{~S} 04$; Ho在 -8.0 至 -8.2 相当于 $85 \mathrm{wt} \%$ 以上的 H2S04。故可推断氟化修饰酸性树脂用作丁烯水合反应的 催化剂将具有较好的催化反应性能。

\section{3. 多孔磺酸氟化树脂催化剂的水合反应性能}

磺酸树脂水热稳定性与其水合反应的催化性能直 接相关, 通过 $200^{\circ} \mathrm{C}$ 水热处理的结果表明大孔磺酸树脂、 凝胶磺酸树脂的水热稳定性相当, 多孔磺酸树脂水热稳 定性略高于普通大孔磺酸树脂; 多孔氯化磺酸树脂、多 
孔氟化磺酸树脂水热稳定性明显优于大孔磺酸树脂和 凝胶磺酸树脂。同时, 通过对氟化修饰树脂用于丁烯水 合反应的催化性能进行反应评价比较可见, 在氯化和氟 化修饰后苯乙烯树脂固体酸的丁烯水合反应活性逐步 明显提高, 选择性也稍有增加, 氟化修饰后树脂丁烯转 化率大于 $13 \%$ 和选择性丁醇大于 $98 \%$; 这就进一步弄清了 树脂酸性与其丁烯水合反应活性和选择性之间的内在 相互关系。

表3 酸性树脂催化剂的水热稳定性与水合反应性能评价。

\begin{tabular}{llll}
\hline 催化剂 & $\begin{array}{l}\text { 酸量 (处理前/处理后) } \\
\mathrm{mmol} / \mathrm{g}\end{array}$ & $\begin{array}{l}\text { 丁烯转化 } \\
\text { 率 \% }\end{array}$ & $\begin{array}{l}\text { 丁醇选择 } \\
\text { 性 \% }\end{array}$ \\
\hline 多孔磺酸树脂 & $4.9 / 4.2$ & 9.8 & 97.9 \\
$\begin{array}{l}\text { 多孔氯化磺酸 } \\
\text { 树脂 }\end{array}$ & $3.8 / 3.6$ & 11.6 & 98.2 \\
$\begin{array}{l}\text { 多孔氟化磺酸 } \\
\text { 树脂 }\end{array}$ & $4.1 / 4.0$ & 13.3 & 99.1 \\
大孔磺酸树脂 & $4.6 / 3.9$ & 6.1 & 97.3 \\
凝胶磺酸树脂 & $4.8 / 4.1$ & 5.4 & 96.0 \\
\hline
\end{tabular}

注: 在反应温度 $165^{\circ} \mathrm{C}$, 水与丁烯比 $2 \mathrm{~mol} / \mathrm{mol}$, 空速WHSV1.0 h-1条件 下。

为了提高树脂固体酸催化剂在烷基化反应过程中水 /丁烯的实际反应比率, 但不增加整个反应过程的水/丁 烯原料比, 拟采用多个床层的多段固定床反应器工艺, 将丁烯原料均匀分成几个部分从每个装填催化剂的每段 床层分开进料, 使每个床层催化剂接触的水/丁烯反应物 料比大幅度提高, 以减少丁烯齐聚副反应的发生与大分 子焦油沉积物的形成, 提高催化剂的水合选择性与反应 稳定性, 并且水过量也可提高仲丁醇SBA的选择性。由表 4 结果可见, 随着填充床层的增加, 催化剂反应接触的水 /丁烯反应物料比的提高, 丁烯转化率和丁醇选择性明显 上升。

表4 反应工艺对催化反应结果的影响。

\begin{tabular}{llll}
\hline 催化剂装填 & $\begin{array}{l}\text { 丁烯单程转化 } \\
\text { 率 }\end{array}$ & 丁醇选择性 \% & $\begin{array}{l}\text { 丁 醇 单 程 收 } \\
\text { 率 \% }\end{array}$ \\
\hline 普通固定床 & 12.8 & 98.3 & 12.6 \\
二段床层 & 13.7 & 98.6 & 13.5 \\
四段床层 & 14.3 & 98.9 & 14.1 \\
\hline
\end{tabular}

注: 在反应温度 $165^{\circ} \mathrm{C}$, 水与丁烯比 $2 \mathrm{~mol} / \mathrm{mol}$, 空速WHSV1.0 h-1条件 下。

采用制备树脂催化剂与配套多段固定床反应工艺, 在 反应温度 $168-174^{\circ} \mathrm{C}$, 水与丁烯比 $2 \mathrm{~mol} / \mathrm{mol}$, 空速WHSV 1.0 $\mathrm{h}-1$ 条件下烯烃直接水合催化反应连续运行评价 $500 \mathrm{~h}$ (仅 在反应 $48 \mathrm{~h}$ 与 $384 \mathrm{~h}$ 各提温 $3^{\circ} \mathrm{C}$ ), 丁烯转化率达到 $15 \mathrm{wt} \%$ 以 上、脂肪醇产物选择性 $99 \mathrm{wt} \%$ 以上, 由图2结果表明催化 剂具有丁烯直接水合催化反应稳定性, 综合性能指标超过 国内外同类清洁合成工艺的技术水平。

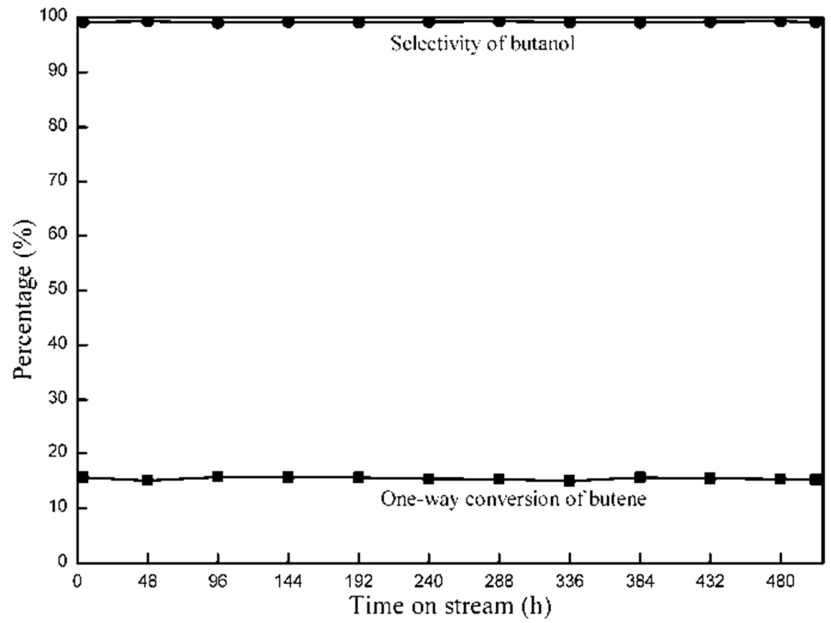

图2 杂化催化剂的丁烯直接水合催化反应稳定性结果。

\section{4. 结论}

采用经碳链脂肪酸改性处理的纳米氧化铝为无机致 孔剂的硬模板支撑造孔方法, 以悬浮聚合方式合成了高比 表面积的多孔苯乙烯树脂颗粒; 以浓硫酸与 $50 \mathrm{wt} \%$ 发烟硫 酸混合物为磺化剂进行酸功能化制备的催化剂酸量可达 4. $9 \mathrm{mmol} / \mathrm{g}$.

采用稀释氟气进行氟化修饰后的苯乙烯树脂固体酸 酸强度Ho显著上升, 可达-8.6, 其酸性适用于丁烯水合反 应的催化剂。

采用四段装填方式固定床反应工艺, 提高反应接触的 水/丁烯反应物料比, 丁烯转化率和丁醇选择性明显上升。

在反应温度 $168-174^{\circ} \mathrm{C}$, 水与丁烯比 $2 \mathrm{~mol} / \mathrm{mol}$, 空速 WHSV1. $0 \mathrm{~h}-1$ 条件下烯烃直接水合催化反应的丁烯转化率 达到 $15 \mathrm{wt} \%$ 以上、脂肪醇产物选择性 $99 \mathrm{wt} \%$ 以上, 并具有 较好的反应稳定性。

\section{致谢}

本文为江苏省科技型企业技术创新资金项目（项目编 号BC2014126）的阶段性成果之一。

\section{参考文献}

[1] 崔锡红, 曾群英, 李吉辉, 赵光辉, 混合碳四中丁烯 -1 的分离 技术及综合利用 $[J], 2011,11: 33-37$ 。

[2] 朱青芳.甲乙酮的合成方法及生产消费情况. 医药化工 [J], 2007 (3) : 33 - 37。

[3] 何英华, 王苑, 杨玉和, 等。C4烃资源综合利用技术进展 [J], 化工技术与开发，2012, 41 (3) :28-34。

[4] 高会元, 国内外甲乙酮生产工艺技术进展 $[\mathrm{J}]$, 化工进展, 2001, 20 (11) : 31-34。 
［5］李亚军,华贲, 李国庆. 我国液化石油气资源的优化利用 $[J]$. 石油炼制与化工, 2005, 36 (3) : 46-50。

[6] 王国强, 碳四烃综合利用研究 $[\mathrm{J}]$, 中国石油和化工标准与 质量, 2014(10) : 16-17。

［7］董炳利, 周金波, 王燕飞, 等. 碳四烃中异丁烯水合制叔丁 醇技术进展 $[J]$ ，应用化工，2012, 41 (4):693-696。

[8] Kharitonov A P, Kharitonov L N, TaegeR. Direct fluorination of polymer vessels and membranes:
Enhancement of barrier and gas separation properties [J] . ActualiteChimique, 2006, 301: 130-134.

[9] Lagow R J, Margrave J L. The controlled reactionof hydrocarbon polymers with elemental fluorine [J].Polym Lett, 1974, 12: 177-184.

[10］王金明, 刘文飞, 氟化树脂催化异丁烯水合反应研究 $[J]$, 化 学工业与工程, 2010, 27:214-218。 